\title{
A qualitative study to explore the experiences of university students which influence eating behaviours when living away from home
}

\author{
A.A. Hafiz, A.M. Gallagher and A.J. Hill \\ School of Biomedical Sciences and Northern Ireland Centre for Food and Health, Ulster University, Coleraine BT52 \\ $1 S A, U K$
}

The transition from post-primary school to tertiary education has been recognised as a vulnerable period regarding the development of unhealthy eating behaviours of students who leave home and move to university ${ }^{(1)}$. Many students struggle to adapt a healthy lifestyle as they, become more independent, are influenced by new peers, have limited finances ${ }^{(2,3)}$. Thus, dietary behaviours of university students may have long-term health implications lasting well into adulthood ${ }^{(4)}$. The aim of this qualitative study was to explore the eating behaviours and experiences of university students in Northern Ireland who regularly live away from home.

Full-time students living away from home for a minimum of 4 nights each week were recruited following a university wide e-mail. European and non-European students, including undergraduate and postgraduate students in all study disciplines/subjects who responded, were invited to participate in a facilitated group discussion using a semi-structured interview schedule. All group discourse was audio-taped, transcribed verbatim and analysed using thematic analysis for common themes. Data were analysed using NVivo 10.

Forty two students ( $n 27$ male; 15 female), both undergraduate $(n 17)$ and postgraduate $(n 25)$ students, participated in 8 facilitated discussions; mean age 23 (SD 5.3) years mean BMI 24.5 (SD 4.6) kg/m². Three key themes were identified to influence eating behaviours. Firstly, nutritional awareness and knowledge: lack of cooking and menu preparation skills. Secondly, personal factors: time and convenience, finance, past habits strength, peer influence and perceived enjoyment. Finally, physical factors: food availability, accessibility, cooking facilities, exam stress/pressures. These key themes together with student recommendations and ideas arising from this focus group work are being used to facilitate the development of an effective and tailored intervention programme aimed at influencing eating behaviours in university students living away from home in Northern Ireland.

This study was carried out with financial support from the Royal Embassy of Saudi Arabia.

1. Kelly NR, Mazzeo SE, Bean MK (2013) J Nutr Educ Behav 45, 304-313.

2. Bray SR, Kwan MYW (2006) J Am Coll Health 55, 77-82.

3. Strong KA, Parks SL, Anderson E et al. (2008) J Am Diet Assoc 108, 1708-1715.

4. Deliens T, Clarys P, De Bourdeaudhuij I et al. (2014) BMC Public Health 14, 53. 